\title{
ANÁLISE DE PADRÕES TÉRMICOS RELACIONADOS AOS NINHOS DE ESPUMA DE LEPTODACTYLUS LABYRINTHICUS SPIX, 1824
}

\author{
FERNANDES, Marcelo Santos ${ }^{1}$ \\ BINO FILHO, Marcos Antonio Tosta ${ }^{2}$ \\ SILVA, Rafael Carvalho da ${ }^{2}$
}

\begin{abstract}
RESUMO: A família Leptodactylidae depositam seus ovos em ninhos de espuma, que dentre outras funções, poderiam participar da manutenção de temperaturas adequadas para o desenvolvimento de ovos, embriões e larvas. O trabalho estudou as variações térmicas entre os ninhos de oviposição de Leptodactylus labyrinthicus e ar externo. Foram realizadas medidas de temperatura do ar, a $10 \mathrm{~cm}$ e do fundo da espuma para 4 ninhos nos seguintes períodos: $10 \mathrm{~h} ; 14 \mathrm{~h} ; 18 \mathrm{~h}$ e $22 \mathrm{~h}$ horas de um único dia. As temperaturas do ar mostraram-se significativamente acima daquelas do fundo dos ninhos, exceto para o período das $22 \mathrm{~h}$ horas, mostrando-se iguais. As temperaturas do fundo não diferiram entre si para os períodos das $12 \mathrm{he} 22 \mathrm{~h}$ horas. Conclui-se que os ninhos de espuma de L. labyrinthicus mantiveram suas temperaturas internas a valores abaixo dos atmosféricos externos.
\end{abstract}

Palavras-Chave: Temperatura do ar. Temperatura do fundo dos ninhos. Reflexão de luz. Bolhas de ar.

SUMMARY: The Leptodactylidae family lay their eggs in foam nests, which, among other functions, could participate maintaining appropriate temperatures for the development of eggs, embryos and larvae. The work studied the thermal variations between the nests of oviposition of Leptodactylus labyrinthicus and outside air. Measurements of air temperature at $10 \mathrm{~cm}$ above and the bottom of the foam to four nests were conducted during the following periods: $10 \mathrm{~h} ; 14 \mathrm{~h} ; 18 \mathrm{~h}$ and $22 \mathrm{~h}$ hours of a single day. Air temperatures were significantly above those of the bottom of the nest, except for the period from $22 \mathrm{~h}$, being equal. The temperatures of the background did not differ for the periods 12 hand $22 \mathrm{~h}$ hours. It is concluded that the foam nests of L. labyrinthicus kept their internal values below the external atmospheric temperatures.

Keyword: Air temperature. Bottom temperature of nests. Reflection of light. Air bubbles.

\section{INTRODUÇÃO}

A família Leptodactylidae constitui um grupo de anuros com ampla distribuição e diversidade pelas Américas, apresentando até o momento 201 espécies formalmente descritas (FROST, 2014). Os membros dessa família depositam seus ovos em ninhos de espuma, fator que foi de primordial importância para a sua irradiação em ambientes de savanas (HEYER, 1975; MAXSON; HEYER, 1982). Foram sugeridas diversas funções para a espuma dos ninhos, como, proteção contra dessecação de ovos, embriões e larvas (HEYER, 1969), proteção contra predadores (DOWNIE,1990), fornecimento de oxigênio (SEYMOUR; LOVERIDGE, 1994), manutenção de temperaturas adequadas para o desenvolvimento (DOBKIN; GETTINGER, 1985) e fonte alimentar (TANAKA; NISHIHARA, 1987).

A morfologia desses ninhos, como área de superfície, profundidade e volume, e o local onde são construídos, exibem grande variação tanto inter quanto intraespecífica. Foi observada grande diversidade de formas e dimensões dos ninhos leptodactilideos, talvez pelo tamanho dos indivíduos que constroem os ninhos e pelos comportamentos sexuais de escolha de locais para a construção dos ninhos (KLUGE, 1981). Um baixo valor da razão entre a área superfícial e volume do ninho parace proporcionar maior proteção contra a dessecação e predação desses sítios de oviposição em Physalaemus e Pleurodema (RYAN, 1985; ZINA, 2006).

\footnotetext{
${ }^{1}$ Dr. em Fisiologia. Docente/Coordenador do curso de Ciências Biológicas da FE/Ituverava.

${ }^{2}$ Licenciatura Plena em Ciências Biológicas. FE/Ituverava.
} 
Leptodactylus labyrinthicus (Spix, 1824), conhecidas popularmente com rã-pimenta, apresenta ampla distribuição pelo Brasil, Bolívia e Paraguai, sendo encontrada desde áreas abertas do Centro-oeste, Sul e Sudeste, com prevalência em domínios de Cerrado, até planaltos e bordas do Pantanal e grande importância ecológica, predando insetos, outros anfíbios e roedores. Dotadas de características como corpo robusto, coloração marcante (de marrom-escuro a marrom-claro no dorso, com manchas escuras, de vermelho a alaranjado na região inguinal e parte interna da coxa, de creme-avermelhado a amarelado no ventre), barras brancas verticais no lábio superior e glândulas na região dorso-lateral, apresentam hábitos terrestres e noturnos. Apresentam comportamentos reprodutivos definidos, onde os machos com espinhos córneos nos polegares e no peito vocalizam e têm com as fêmeas, amplexos axilares em lagoas, geralmente em regiões abertas. As desovas, com aproximadamente 2.000 ovos, são colocadas em bacias escavadas pelo macho ou pelo casal, próximas a touceiras de capim, no chão, ou próximos as margens das lagoas. Cerca de $10 \%$ dos ovos são fecundados, servindo o restante com alimento para os girinos que eclodirão (UETANABARO et al, 2008). O presente estudo teve como objetivo analisar as variações térmicas entre os ninhos de oviposição de L. labyrinthicus e seu meio externo. O presente trabalho se justificou pela importância que os estudos referentes a questões ambientais poderia comtribuir para a conservação e preservação das populações de anfíbios em todo o mundo.

\section{MATERIAL E MÉTODO}

\section{Área de Estudo e coleta de dados}

A área de estudo, uma vegetação com características de cerrados ao redor de uma lagoa (latitude $20^{\circ} 21^{\prime} 44.49^{\prime \prime} \mathrm{S}$ e longitude $47^{\circ} 46^{\prime} 25.92^{\prime \prime} \mathrm{W}$ ), está localizada no município de Ituverava (latitude 20²0'30"S e longitude 4747'30" W), a nordeste do estado de São Paulo. O município tem um clima que é considerado do tipo AW - Megatérmico (tropical úmido), característico de savanas tropicais com verão úmido e inverno seco e ameno, com temperaturas dos meses mais frios superiores a $18^{\circ} \mathrm{C}$ (CARRER; GARCIA, 2007).

\section{Coleta de dados}

As coletas de dados foram realizadas entre os mês de outubro e dezembro de 2013, mapeando-se 4 ninhos de espuma de L. labyrinthicus que estavam dispostos as margens da lagoa entre a vegetação. Foram realizadas medidas de temperatura do ar, a $10 \mathrm{~cm}$ e do fundo da espuma, utilizando-se o termômetro Dellt (DT-900; -50 a $200^{\circ} \mathrm{C}$ ). Em cada ninho foram realizadas 4 medidas de temperatura em diferentes períodos de um único dia (às 10h horas; às 14h horas, às 19:00 horas e às 22h horas). O presente trabalho esteve legalmente amparado pela Licença permanente para coleta de material zoológico, de número 19125-2, fornecida pelo IBAMA.

\section{Análise dos dados}

Os dados experimentais foram analisados utilizando-se o programa estatístico GraphPad Prism 5. Para se testar possíveis diferenças entre as temperaturas do ar e a do fundo dos ninhos de espuma foi utilizado o teste " $\mathrm{t}$ " de Student, sendo os dados apresentados como média \pm erro padrão médio (SEM), sendo considerados significativos $\mathrm{p} \leq 0,05$ (Zar, 1984).

\section{RESULTADO}

Os ninhos estudados foram encontrados em regiões com grande intensidade de vocalizações, estando esses entre os arbustos e plantas herbáceas ao redor do lago. Esses ninhos apresentaram 
profundidade média de $11 \pm 0,25 \mathrm{~cm}$, com a presença de muitos ovos.

A espuma mostrou-se em cor branca brilhante e viscosa, mostrando refletir a luz solar e proteger os ovos contra formigas que tentavam predá-los sem sucesso. Observou-se que alguns ninhos estavam mais hidratados do que outros. Alguns ninhos apresentavam a superfície na forma de uma crosta enrijecida, parecendo afastar a presença de formigas. Um dos ninhos estava com a espuma baixa e diluída com girinos no fundo, que pareciam respirar na superfície e se alimentar da espuma que ainda restava. Durante o dia ficavam no fundo dos ninhos e subiam de forma intermitente à superfície, evidenciando a necessidade de trocas gasosas aéreas, possivelmente pela presença já de pulmões.

As temperaturas do ar às $10 \mathrm{~h}$ horas $\left(28.98 \pm 0.74{ }^{\circ} \mathrm{C}\right)$ mostraram diferenças significativas quando comparadas às temperaturas do fundo dos ninhos $\left(23.98 \pm 0.33^{\circ} \mathrm{C}\right)$, como vista na figura 1.

Figura 1. Temperaturas $\left({ }^{\circ} \mathrm{C}\right)$ do ar externo e do fundo dos ninhos de espuma de Leptodactylus labyrinthicus, medidas às 10: horas. Média $\pm \mathrm{SEM} ; \mathrm{n}=4$. Média $\pm \mathrm{SEM} ; \mathrm{n}=4$. Teste " $\mathrm{t}$ " de Student. (*) representa as diferenças significativas entre as temperaturas do ar e do fundo dos ninhos $(\mathrm{p}<0,05)$.

\section{HORAS}

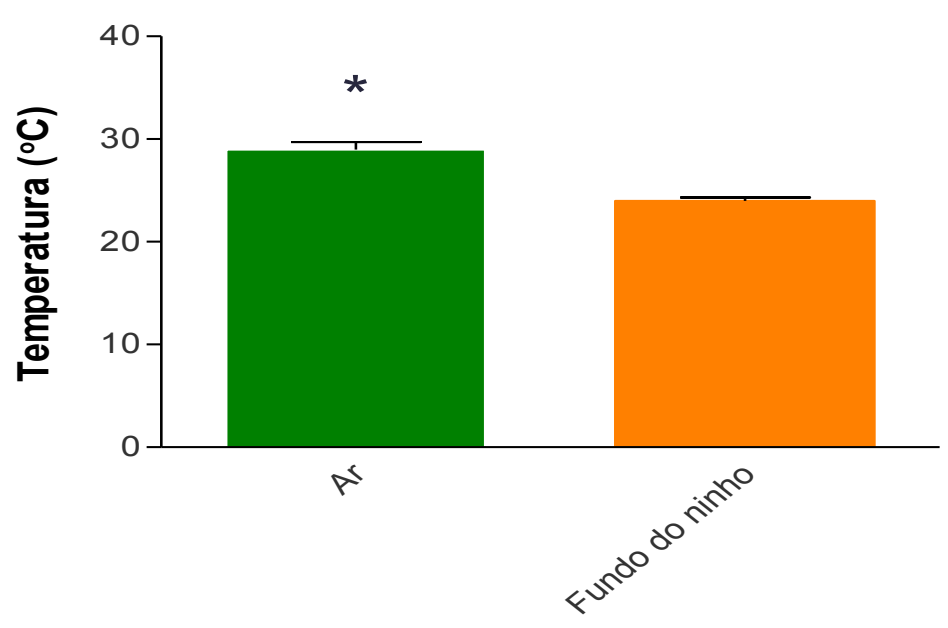

O mesmo ocorreu com as temperaturas medidas às $14 \mathrm{~h}$ horas $\left(39.38 \pm 2.69 \mathrm{e} 24.63 \pm 0.48^{\circ} \mathrm{C}\right) \mathrm{e}$ às $18 \mathrm{~h}$ horas $\left(31.10 \pm 0.58\right.$ e $\left.25.90 \pm 0.36^{\circ} \mathrm{C}\right)$, vistas nas figuras 2 e 3 .

Figura 2. Temperaturas $\left({ }^{\circ} \mathrm{C}\right)$ do ar externo e do fundo dos ninhos de espuma de Leptodactylus labyrinthicus, medidas às 14: horas. Média $\pm \mathrm{SEM} ; \mathrm{n}=4$. Média $\pm \mathrm{SEM} ; \mathrm{n}=10$. Teste " $\mathrm{t}$ " de Student. $\left(^{*}\right)$ representa as diferenças significativas entre as temperaturas do ar e do fundo dos ninhos $(p<0,05)$.

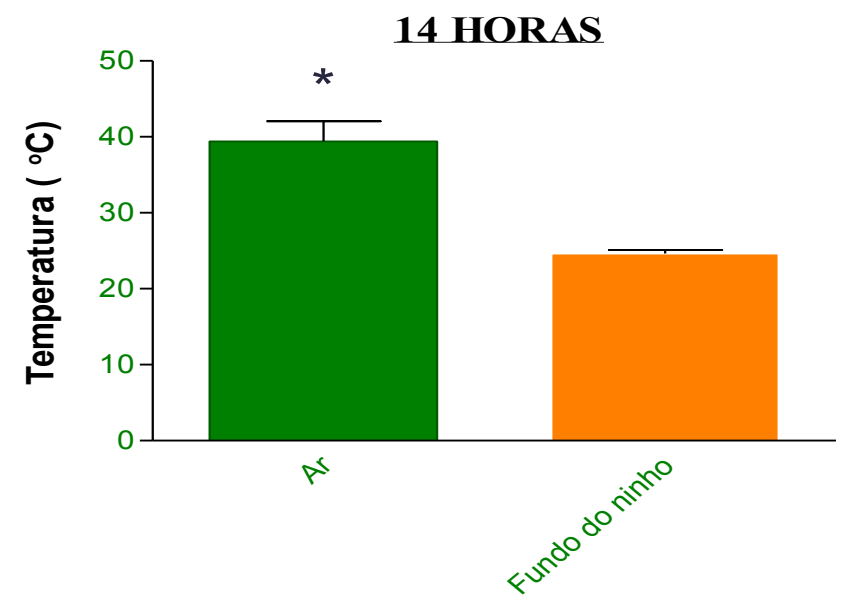


Figura 3. Temperaturas $\left({ }^{\circ} \mathrm{C}\right)$ do ar externo e do fundo dos ninhos de espuma de Leptodactylus labyrinthicus, medidas às 18: horas. Média $\pm \mathrm{SEM} ; \mathrm{n}=4$. Média \pm SEM; $\mathrm{n}=10$. Teste " $\mathrm{t}$ " de Student. (*) representa as diferenças significativas entre as temperaturas do ar e do fundo dos ninhos $(p<0,05)$.

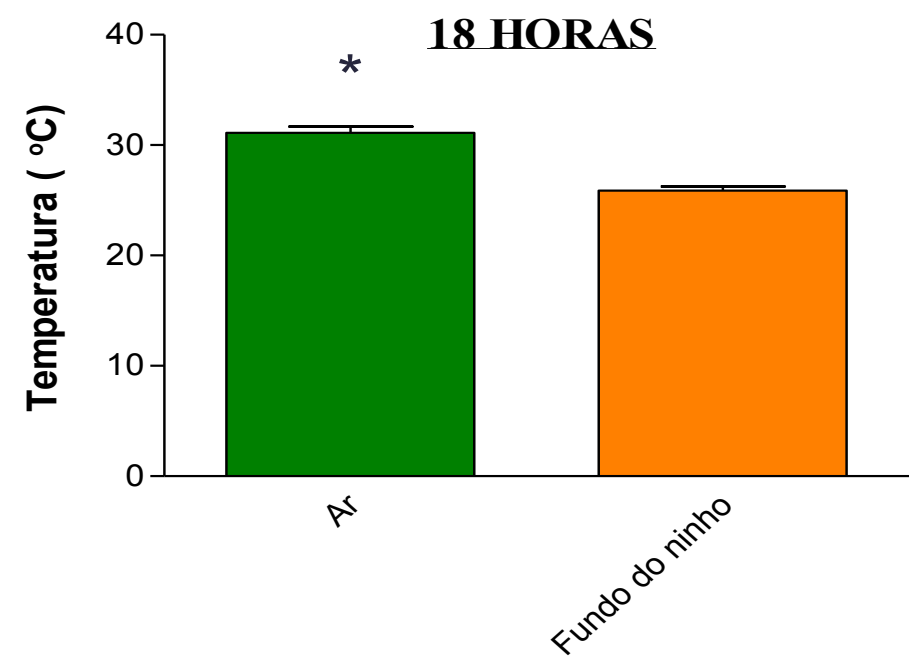

As temperaturas medidas para o ar às $22 \mathrm{~h}$ horas $\left(25.70 \pm 1.07^{\circ} \mathrm{C}\right.$ e $\left.25.38 \pm 0.21^{\circ} \mathrm{C}\right)$ não mostram diferenças estatisticamente significativas (Figura 4).

Figura 4. Temperaturas $\left({ }^{\circ} \mathrm{C}\right)$ do ar externo e do fundo dos ninhos de espuma de Leptodactylus labyrinthicus, medidas às 22: horas. Média $\pm \mathrm{SEM} ; \mathrm{n}=4$. Média $\pm \mathrm{SEM} ; \mathrm{n}=10$. Teste " $\mathrm{t}$ " de Student. (*) representa as diferenças significativas entre as temperaturas do ar e do fundo dos ninhos $(p<0,05)$.

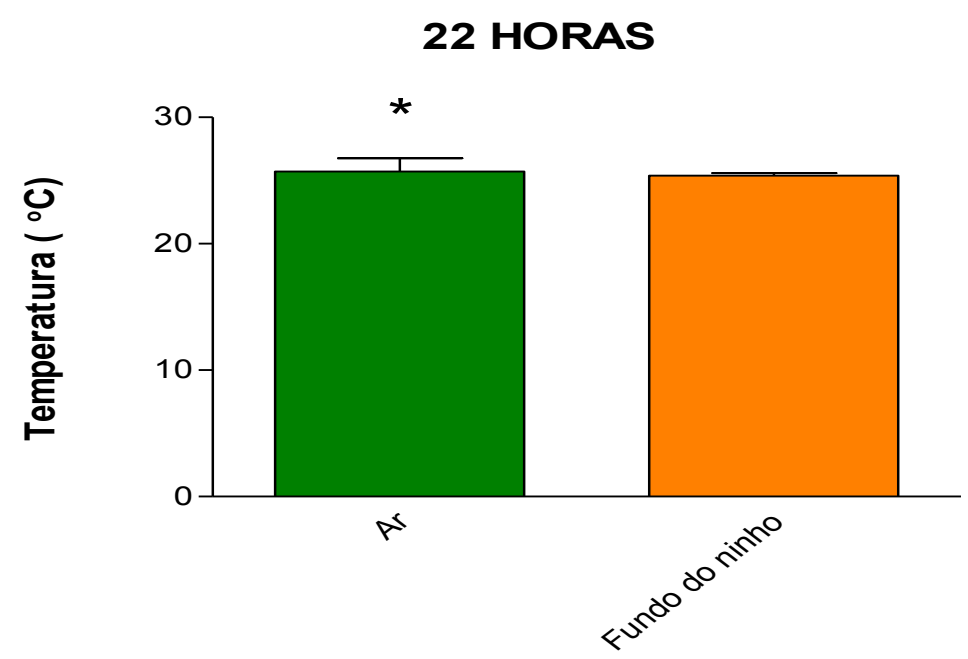

As variações nas temperaturas do ar entre os horários $12 \mathrm{he} 22 \mathrm{~h}$ horas $\left(39.38 \pm 2.69^{\circ} \mathrm{C}\right.$ e $25.70 \pm$ $1.07{ }^{\circ} \mathrm{C}$ ) foram consideradas significativamente diferentes, o que não se observou para as temperaturas do fundo dos ninhos para os mesmos horários $\left(24.63 \pm 0.48^{\circ} \mathrm{C}\right.$ e $\left.25.38 \pm 0.21^{\circ} \mathrm{C}\right)$.

\section{DISCUSSÃO}

As temperaturas do ar, acima de $10 \mathrm{~cm}$ dos ninhos, mostram-se superiores àquelas do fundo da espuma para os horários de $10 \mathrm{~h}$ horas $\left(28.98 \pm 0.74{ }^{\circ} \mathrm{C} / 23.98 \pm 0.33^{\circ} \mathrm{C}\right), 14 \mathrm{~h}$ horas $\left(39.38 \pm 2.69^{\circ} \mathrm{C} \mathrm{e}\right.$ 
$\left.24.63 \pm 0.48^{\circ} \mathrm{C}\right)$ e $18 \mathrm{~h}$ horas $\left(31.10 \pm 0.58^{\circ} \mathrm{C}\right.$ e $\left.25.90 \pm 0.36^{\circ} \mathrm{C}\right)$, períodos esses em que o calor era mais intenso. $\mathrm{O}$ mesmo não se observou para o horário das $22 \mathrm{~h}$ horas $\left(25.70 \pm 1.07^{\circ} \mathrm{C}\right.$ e $\left.25.38 \pm 0.21^{\circ} \mathrm{C}\right)$.

Apesar das variações nas temperaturas do ar entre os horários de $12 \mathrm{he} 22 \mathrm{~h}$ horas $\left(39.38 \pm 2.69^{\circ} \mathrm{C}\right.$ e $25.70 \pm 1.07^{\circ} \mathrm{C}$ ), as temperaturas do fundo dos ninhos permaneceram praticamente as mesmas para o período em questão $\left(24.63 \pm 0.48^{\circ} \mathrm{C}\right.$ e $\left.25.38 \pm 0.21^{\circ} \mathrm{C}\right)$.

A alta incidência dos raios solares sobre os ninhos na área de estudo durante todo o dia e a vegetação arbustiva de cerrado, que inclusive se apresentava com alto nível de degradação, poderiam acarretar em aumento da temperatura desses sítios de oviposição, mas isso não foi observado. Diferentemente do período diurno, as noites apresentam temperaturas do ar mais amenas.

Os dados desse estudo corroboram registros obtidos por Shepard e Caldwell (2005), que mostraram que ninhos Leptodactylus labyrinthicus apresentavam temperaturas internas inferiores as do ar ao redor desses $\left(34.2^{\circ} \mathrm{C}\right.$ para o ar, $31.2 \pm 1.9^{\circ} \mathrm{C}$ para a superficie e $28.6 \pm 0.5^{\circ} \mathrm{C}$ para o fundo).

Da mesma forma, tais dados corroboram os estudos de Rodrigues e colaboradores (2012), que ao estudar Physalaemus cuvieri observaram temperaturas do ar ao redor dos ninhos maiores do que as da superfície $\left(\mathrm{T}_{\mathrm{S}}=28,3 \pm 0,36^{\circ} \mathrm{C}\right)$, da porção central $\left(\mathrm{T}_{\mathrm{C}}=25,8 \pm 0,26^{\circ} \mathrm{C}\right)$ e do fundo $\left(\mathrm{T}_{\mathrm{F}}=24,2 \pm 0,22^{\circ} \mathrm{C}\right)$. Apesar dos ninhos de L. labyrinthicus serem mais volumosos do que os de P. cuvieri, são construídos de forma muito similares (ZINA; HADDAD, 2005).

Espécie de ambientes mais frios, como é o caso de Physalaemus pustulosus mostraram temperaturas no interior dos ninhos mais elevadas do que as temperaturas do ar (DOBKIN; GETTINGER, 1985).

As temperaturas dos sítios de oviposição de anfíbios dependem de fatores, como, a hora do dia, a duração da irradiação solar, a presença de nuvens e sombras feitas pela vegetação sobre os ninhos, características do solo e a morfologia dos ninhos (WELLS, 2007).

A morfologia dos ninhos e sua espuma parecem ter correlações importantes entre as trocas térmicas desse com seu meio circundante (CARDOSO, 1981). A baixa relação área/volume poderia reduzir as trocas de calor entre os ninhos de espuma e o ar. Apesar de não terem sido realizadas medidas com essas variáveis para L. labyrinthicus, nota-se que os ninhos dessa espécie possui aberturas que são proporcionalmente menores do seu volume. Os ninhos de $P$. cuvirei mostraram uma baixa relação área/volume apresentando em média um volume 8,3 vezes maior que a área de abertura (RODRIGUES et al., 2014) . Ryan (1985) e Zina (2006) também observaram baixa relação entre área e volume para ninhos de espuma de outras espécies de Physalaemus e Pleurodema.

Foi observado por Rodrigues e colaboradores (2012) que a condução térmica entre o ar acima dos ninhos e seu fundo $\left(\mathrm{Q}_{\mathrm{a}-\mathrm{f}}=9,5.10^{-3} \pm 0,002 \mathrm{~W}\right)$ seria 2,5 vezes maior do que a condução térmica entre a superfície e o fundo dos ninhos $\left(\mathrm{Q}_{\mathrm{s}-\mathrm{f}}=3,8 \cdot 10^{-3} \pm 0,001 \mathrm{~W}\right)$, fato que corroboraria a hipótese de que a espuma teria funcão na redução das trocas de calor. Além disso as superfícies dos ninhos voltadas para o solo poderiam atuar como dissipadores de calor, pela razão desse meio conter água, que apresenta alto calor específico (HALLIDAY et al, 2012).

A espuma branca de brilho intenso, que chega ofuscar a visão, presente na abertura dos ninhos, evidencia a reflexão da luz durante os dias ensolarados. A baixa condutividade térmica do ar (HALLIDAY et al, 2012) presente em pequenas bolhas na espuma poderia ser um fator importante na redução da temperatura no sentido vertical dos ninhos (EINUM et al., 2002; EVANS et al., 1996).

\section{CONCLUSÃO}

Conclui-se que os ninhos de espuma de L. labyrinthicus mantiveram suas temperaturas internas a 
valores abaixo dos atmosféricos externos, pelo menos durante os períodos analisados, fortalecendo a hipótese de que esses sítios de oviposição têm papel vital na manutenção de condições térmicas adequadas ao desenvolvimento de ovos, embriões e larvas.

\section{REFERÊNCIAS}

CARDOSO, A.J. 1981. Biologia e sobrevivência de Physalaemus cuvieri Fitz, 1826 (Amphibia, Anura), na natureza. Cien. Cult, v.33, p. 1224-1228.

CARRER, T. T.; GARCIA, A.. CLASSIFICAÇÃO CLIMÁTICA PARA A CIDADE DE ITUVERAVA /SP. Nucleus, Ituverava, v. 4, n. 1, oct. 2007. ISSN 1982-2278. Disponível em:

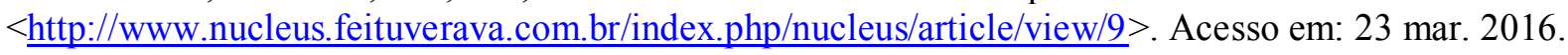

DOBKIN, D.S.; GETTINGER, R.D. Thermal aspects of anuran foam nests. Journal of Herpetology, p. 271-275, 1985.

DOWNIE, J.R. Functions of the foam-nesting leptodactylids: Anti-predator effects of Physalaemus pustulosus foam. Herpetological journal v.1, n.11, p. 501-503, 1990.

EINUM, S.; HENDRY, A.P.; FLEMING, I.A. Egg-size evolution in aquatic environments: Does oxygen availability constrain size? Proceedings of the Royal Society of London. Series B: Biological Sciences, v.269, n.1507, p.2325-2330, 2002.

EVANS, M.; YABER, C.; HERO, J.M. Factors influencing choice of breeding site by Bufo marinus in its natural habitat. Copeia. p.904-912, 1996.

FROST, D.R. Amphibian Species of the World: an Online Reference. Version 6.0 (Date of access). Electronic Database accessible at http://research.amnh.org/herpetology/amphibia/index.html . New York, USA: American Museum of Natural History, 2014.

HALLIDAY D, RESNICK D, WALKER R. Fundamentos de física: gravitação, ondas e termodinâmica, 9. ed. Rio de Janeiro: LTC, 2012.

HEYER, W.R. The adaptive ecology of the species groups of the genus Leptodactylus (Amphibia, Leptodactylidae). Evolution, p.421-428, 1969.

HEYER, W.R A preliminary analysis of the intergeneric relationships of the frog family Leptodactylidae. Smithsonian Contributions to Zoology, v.199, p.1-55, 1975.

KLUGE, A.G. The life history, social organization, and parental behaviour of Hyla resenbergi Boulenger, a nest-building gladiator frog. Misc.Mus.Zool. Univ. Michigan, 1981.

MAXSON, L. R., HEYER, W. R. Leptodactylid frogs and the Brasilian Shield: an old and continuing adaptive relationship. Biotropica, p.10-15, 1982.

RODRIGUES, C.L.; GUILHERME, F.P.F.; FERNANDES, M.S. Análise de fatores abióticos associados aos ninhos de espuma de Physalaemus cuvieri Fitzinger, 1826. In: CONGRESSO DE INICIAÇÃO CIENTÍFICA, Anais... 2012.

RYAN, M.J. The Túngara Frog. Chicago, The University of Chicago, 1985.

SEYMOUR, R.S.; LOVERIDGE, J.P. Embryonic and larval respiration in the arboreal foam nests of the African frog Chiromantis xerampelina. Journal of Experimental Biology v.197, n. 1, p. 31-46, 1994. 
SHEPARD, D.B.; CALDWELL, J.P. 2005. From foam to free-living: ecology of larval Leptodactylus labyrinthicus. Copeia, v.4, p. 803-811, 2005.

TANAKA, S.; NISHIHIRA, M. 1987. Foam nest as a potential food source for anuran larvae: A preliminary experiment. Journal of Ethology, v. 5, n. 1, p. 86-88, 1987.

UETANABARO, M. Field Guide to the anurans of the pantanal and surrounding. Cerrado. Campo Grande: UFMS , 2008. 196 p.

ZAR, J.H. Biostatistical Analysis. 2. ed. Englewood Cliffs: Prentice-Hall, 1984.

ZINA, J. Communal nests in Physalaemus pustulosus (Amphibia: Leptodactylidae): Experimental evidence for female oviposition preferences and protection against desiccation. Amphibia Reptilia v.27, n.1, p. 148, 2006.

ZINA, J.; HADDAD, C.F.B. Reproductive activity and vocalizations of Leptodactylus labyrinthicus (Anura: Leptodactylidae) in Southeastern Brazil. Biota Neotropica, v.5, n.2, 2005. Disponível em: http://www.biotaneotropica.org.br/v5n2/pt/abstract?article+BN00605022005

WELLS, K.D. The ecology and behavior of amphibians. Chicago: University of Chicago Press, 2007. 
